\title{
THE FIVE-FACTOR MODEL APPROACH TO HYPERTENSIVE PATIENTS: A CASE CONTROL STUDY
}

\author{
EVA-KATALIN LAKATOS ${ }^{1}$, AUREL NIRESTTEAN ${ }^{2}$ \\ 1,2 “George Emil Palade” University of Medicine, Pharmacy, Science and Technology of Târgu Mureș, 'Emergency County Hospital Satu-Mare, \\ ${ }^{2}$ Clinic of Psychiatry II, Târgu Mureș
}

Keywords: hypertension, Five-Factor Model, personality dimensions, neuroticism, emotional stability
Abstract: Objectives: The aim of this case-control pilot study was to examine whether there are differences in personality characteristics between hypertensive and normotensive individuals in terms of the Five-Factor Model (FFM). Methods: 71 individuals with primary hypertension were compared with 84 normotensive individuals on the DECAS personality inventory, assessing the five basic dimensions of personality according to the FFM. Results: Agreeableness and emotional stability were associated with hypertension ( $p-0,0001$ for both). Hypertensive individuals showed low and very low agreeableness and emotional stability whereas normotensives scored medium and high in these two dimensions. Lower emotional stability was a risk factor for hypertension (OR=4.51, CI 95\%=2.189.35). There were no significant differences in openness, extraversion and consciousness between hypertensives and normotensives ( $p>0.05$ ). Conclusion: Individuals with low emotional stability/high neuroticism have more than fourfold increased risk of developing hypertension.

\section{INTRODUCTION}

Hypertension, the "silent killer", is the leading risk factor for the development of cardiovascular diseases (CVD) and premature death. Approximately 1.39 billion individuals are affected worldwide, with a globally rising prevalence estimated to affect one third of the world's population by 2025, due to the increasing obesity and ageing of the population. Hypertension is considered one of the world's biggest public health problem.(1) Comparably to other Central and East European countries, the prevalence of hypertension in Romania is estimated at $45.1 \%$, while CVD as the result of hypertension is the leading cause of all deaths (62\%).(2)

A number of genetic, physiological and psychosocial risk factors are now recognized in the etiopathogenesis of hypertension, facilitating prevention and improving disease management. $(1,3,4)$ However, the full range of factors contributing to disease development is not known. Despite the evidence that personality has an important role in the development of hypertension $(5,6,7)$, personality is not included into hypertension risk predictor models. Most studies examine the role of personality in the development of CVD, instead of directly exploring the relationship between personality and the development of hypertension, the major CVD risk factor.

Personality can be described by five basic traits/dimensions, known as the Five-Factor Model (FFM) or the Big Five personality traits: N- Emotional Stability/Neuroticism (the tendency to experience negative emotions, sensitive/nervous vs. secure/confident), E-Extraversion (outgoing, energetic vs. solitary/reserved), O-Openness to experience (inventive/curious vs. consistent/cautions), A-Agreeableness (friendly/compassionate vs. challenging/detached), C-Conscientiousness (efficient /organized vs. easy-going/careless).(8,9,10) The FFM is the dimensional approach to describing adaptive and maladaptive personality characteristics. Another approach to determine personality is the bimodal, categorical approach, where an individual can be characterized as having a type D "distressed" or non-Type D personality.(11) The core traits of the Type D personality, negative affectivity (NA) and social inhibition (SI), could be easily described in terms of the FFM of personality, where NA primarily correlates with Neuroticism while SI with Extraversion (negatively) and with Neuroticism.(12) Other concepts and theories regarding personality traits can be integrated into/described by the FFM, making this model the reference system for the description of personality. Type D or "distressed" personality was recognized as a significant negative prognostic factor for patients with CVD, particularly in the case of coronary heart disease, oversighting the potential relationship between personality and hypertension, the most critical and reversible risk factor for CVD.

\section{AIM}

The purpose of the present case-control study therefore was to examine how personality traits, as described in the FFM, differ between normotensive and hypertensive individuals and if personality dimensions could be considered as risk factors for the development of hypertension.

\section{MATERIALS AND METHODS}

Ethical approval and consent to participate

This case-control pilot study was approved by the local Research Ethics Committee (REC NR38/17102012) and was conducted in accordance with the Declaration of Helsinki in 1995 (as revised in Edinburgh 2000). Participants were recruited on a voluntary basis, following written informed consent, at the Emergency Institute for Cardiovascular Diseases and Transplantation Târgu-Mureș.

\section{Participant recruitment}

71 consecutive hypertensive patients were recruited to the case group and 84 non-hypertensive controls to the control group. Recruited participants had to have either a clear diagnosis

${ }^{1}$ Corresponding author: Eva-Katalin Lakatos, Str. Prahova, Nr.1-3, Satu-Mare, România, E-mail: drevalakatos@ yahoo.com, Phone: +40756 203056 Article received on 10.11.2021 and accepted for publication on 02.12.2021 


\section{PUBLIC HEALTH AND MANAGEMENT}

of essential/primary hypertension for the case group or the lack of hypertension for the control group, having an age between 40-65 years, with an educational level of at least 10 classes, with the ability to understand and write the Romanian language and capacity to give informed consent for study participation. Patients with secondary hypertension or with a known mental health condition (such as addiction, anxiety, depression, posttraumatic stress, bipolar disorder, personality disorder, obsessivecompulsive disorder, eating disorder, schizophrenia, any type of dementia) were not included.

\section{Data collection}

The data were prospectively recorded with standard forms, including age, gender, weight, height, physical activity, smoking status (never smoked, former or active smoker) and educational level of each participant. Evaluations and measurements were performed by the same research assistant. Essential arterial hypertension was defined as having a systolic blood pressure (SBP) of $\geq 140 \mathrm{mmHg}$ and/or a diastolic blood pressure (DBP) of $\geq 90 \mathrm{mmHg}$, in the lack of an identifiable cause, which necessitated antihypertensive treatment.(4) For newly diagnosed patients, blood pressure measurements were taken in clinic on three separate occasions. Obesity was graded according to Body Mass Index (BMI) as grade I (BMI: $30.00-34.99 \mathrm{~kg} / \mathrm{m}^{2}$ ), grade II (BMI $35.00-39.99 \mathrm{~kg} / \mathrm{m}^{2}$ ) or grade III (BMI $\geq 40.00$ $\mathrm{kg} / \mathrm{m}^{2}$ ). Patients having moderate physical activity for at least 30 min/day, 5 days/week were considered physically active.(5)

\section{Personality Assessment}

This study used the DECAS Personality Inventory to assess the five basic personality dimensions described by the FFM. This inventory was based on the NEO-PI-R questionnaire, originally developed by Costa and McCrae for the assessment of the five factors and their lower level traits, but it was validated on the Romanian population. The two inventories have a good concurrent validity ranging between 0.57 and 0.81 . The DECAS acronym stands for D-Deschidere (Openness), E-Extraversiune (Extraversion), C-Conștiinciozitate (Conscientiousness), AAgreabilitate (Agreeableness), S-Stabilitate Emoţională (Emotional Stability/Neuroticism). It contains 97 statements requiring a True/False answer. The statements are distributed in 5 content scales according to FFM. The inventory also includes 3 validation scales in order to determine the validity of answers, namely: the social desirability (SD) validation scale, assessing the degree at which individuals defer from their regular behaviour in order to present themselves into a more favourable light; the random answers (RA) validation scale, evaluating the degree at which the questionnaire was filled out at random; and the approval (AP) validation scale, assessing the tendency to choose predominantly "true" or "false" answers, independently of the statement. Raw test scores from the validations scales are transformed into standard $\mathrm{T}$ quotients, with a value over 65 demarking an invalid protocol. Similarly, T quotients under 35 obtained on the AP validation scale indicate an invalid protocol recognizing the tendency of a participant to negatively respond to the questionnaire items. $(15,16)$

\section{Statistical Analysis}

Statistical analysis was performed using the Statistical Package for Social Sciences (SPSS, version 25.0). Data was classified into categorical (dichotomous, nominal) or quantitative variables. Means of frequencies were used to characterize nominal variables. For quantitative data the Kolmogorov-Smirnov test was used to test for normality of distribution and mean \pm standard deviation or median and percentiles were described where appropriate. Independent sample Student's t-tests were run for quantitative variables to determine the significant differences in the means between the hypertensive (cases) and non-hypertensive (controls) groups. The $\mathrm{T}$ quotient results of the different dimensions were quantized into the following intervals: very low: 20.00-34.99; low: 35.00-44.99; medium: 45.00-55.00; high: 56.01-65.99; very high: 66.00-88.00. The frequencies of nominal variables were compared with a Chi-square test. To estimate the strength of associations, the personality dimensions were divided into dichotomous variables according to these optimal cut-off points, (very low and low with $1 /$ very high, high and moderate with 0 ) and adjusted odds ratios (OR) with their $95 \%$ confidence interval (CI) for cases versus control were calculated. The level of statistical significance was set at $\mathrm{p}<0.05$.

\section{RESULTS}

From the 155 recruited participants, 138 passed all three validation scales, having T quotients lower than 65 but higher than 35 on the AP scale, and were included in the data analysis. A valid protocol was obtained for 60 out of $71(84.5 \%)$ cases and for 78 out of $84(92.8 \%)$ controls. The demographic and clinical characteristics of the two participant groups are presented in table no. 1. There were no significant differences between the two participant groups in age and gender.

Table no. 1. Demographic and clinical characteristics of participants

\begin{tabular}{|c|c|c|c|}
\hline 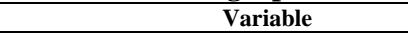 & Hypertensive group (cases) $n=60$ & Normotensive group (controls) n=78 & $p$ value \\
\hline \multicolumn{4}{|c|}{ ( } \\
\hline Male/Female, $\operatorname{nr}(\%)$ & $35(58.3) / 25(41.6)$ & $41(52.5) / 37(47.4)$ & $>0.05$ \\
\hline Age (years) & $55 \pm 7$ & $50 \pm 8$ & $>0.05$ \\
\hline \multicolumn{4}{|l|}{ Smoking status } \\
\hline Current smoker, nr (\%) & $6(10.0)$ & $20(25.6)$ & \multirow{3}{*}{0.001} \\
\hline Former smoker, $\mathrm{nr}(\%)$ & $28(46.7)$ & $10(12.8)$ & \\
\hline Never smoker, nr (\%) & $26(43.3)$ & $48(61.5)$ & \\
\hline \multicolumn{4}{|l|}{ Obesity } \\
\hline Not-obese & $30(50.0)$ & $64(82.0)$ & \multirow{4}{*}{0.001} \\
\hline Grade I, nr (\%) & $23(38.3)$ & $7(8.9)$ & \\
\hline Grade II, nr (\%) & $5(8.3)$ & $6(7.6)$ & \\
\hline Grade III, nr (\%) & $2(3.3)$ & $1(1.2)$ & \\
\hline \multicolumn{4}{|l|}{ Physical activity } \\
\hline$<30 \mathrm{~min} / \mathrm{day}$ & $39(65.0)$ & $48(61.5)$ & \multirow[t]{2}{*}{$>0.05$} \\
\hline$\geq 30 \mathrm{~min} / \mathrm{day}$ & $21(35.0)$ & $30(38.5)$ & \\
\hline \multicolumn{4}{|l|}{ Education } \\
\hline 10 classes/Professional school & $14(23.4)$ & $10(12.8)$ & \multirow{3}{*}{$>0.05$} \\
\hline Secondary school & $27(45.0)$ & $35(45.4)$ & \\
\hline University & $19(31.6)$ & $33(42.8)$ & \\
\hline \multicolumn{4}{|l|}{ Personality dimensions ( $\mathrm{T}$ quotients) } \\
\hline Openness & $47.62 \pm 9.39$ & $49.92 \pm 10.63$ & 0.19 \\
\hline Extraversion & $49.09 \pm 11.08$ & $51.22 \pm 10.66$ & 0.25 \\
\hline Agreeableness & $46.93 \pm 9.04$ & $48.35 \pm 7.79$ & 0.32 \\
\hline Consciousness & $48.96 \pm 12.36$ & $47.39 \pm 10.19$ & 0.41 \\
\hline Emotional Stability & $43.57 \pm 7.22$ & $49.35 \pm 9.54$ & 0.0001 \\
\hline
\end{tabular}


PUBLIC HEALTH AND MANAGEMENT

Table no. 2. Agreeableness and emotional stability as potential risk factors for hypertension

\begin{tabular}{|c|c|c|c|}
\hline \multicolumn{2}{|c|}{} & $\begin{array}{c}\text { Normotensive group (controls) } \\
\text { n=78 }\end{array}$ & $\begin{array}{c}\text { Hypertensive group (cases) } \\
\text { n=60 }\end{array}$ \\
\hline \multirow{4}{*}{$\begin{array}{c}\text { Agreeableness } \\
\text { p-0.0001 }\end{array}$} & Very high, n-\% & $0-0.0 \%$ & $3-5.0 \%$ \\
\cline { 2 - 4 } & High, n-\% & $15-19.2 \%$ & $4-6.7 \%$ \\
\cline { 2 - 4 } & Medium, n-\% & $37-47.4 \%$ & $31-51.7 \%$ \\
\cline { 2 - 4 } & Low, n-\% & $26-33.3 \%$ & $16-26.7 \%$ \\
\hline \multirow{3}{*}{$\begin{array}{c}\text { Emotional Stability } \\
\text { p-0.0001 }\end{array}$} & Very high, n-\% & $0-0.0 \%$ & $6-10.0 \%$ \\
\cline { 2 - 4 } & High, n-\% & $2-2.6 \%$ & $0-0.00 \%$ \\
\cline { 2 - 4 } & Medium, n-\% & $21-26.9 \%$ & $6-10.0 \%$ \\
\cline { 2 - 4 } & Low, n-\% & $27-34.6 \%$ & $11-18.3 \%$ \\
\cline { 2 - 4 } & Very low, n-\% & $23-37.1 \%$ & $39-65.0 \%$ \\
\hline
\end{tabular}

To highlight the statistical differences between the mean quotients of the five personality dimensions for the cases and the controls, we applied the Student's t-test. Statistically significant difference was found for emotional stability, where the mean quotient value was greater for the control group compared to the cases, 49.3 versus 43.5 (p-0.0001). Other personality dimensions showed comparable mean values. We have also examined if there is a difference in the lifestyle (smoking status, obesity and physical activity,) and educational level of hypertensive versus normotensive individuals. As expected, hypertensive patients had significantly higher obesity levels and positive smoking histories (table no. 1).

To track whether a personality component constitutes a potential risk factor for hypertension, the Chi-square test was applied. Statistically significant differences were found between the case and control groups for agreeableness (p-0.0001) and emotional stability (p-0.0001), with no significant associations for openness, extraversion or conscientiousness. High agreeableness was observed in the control group (19.2\%) while very low levels were detected for the cases (10.0\%). Respectively, medium (26.9\%) and high (34.6\%) emotional stability was observed in the control group but low for the cases $(65.0 \%)$ (table no. 2). In order to perform risk calculation (OR), the manifestation levels of the different dimension were dichotomized, where very low and low levels were coded with 1 , meaning risk, while medium, high and very high levels were coded with 0 , meaning without risk. Thus, the following statistics was obtained for agreeableness: OR: 1.15 (95\% CI: 0.57-2.34), but without statistical significance: p-0.72, and for emotional stability: OR: 4.51 (95\% CI: 2.18-9.35), with statistical significance: $\mathrm{p}-0.0001$, showing that low or very low emotional stability is a potential risk factor for hypertension

\section{DISCUSSIONS}

This case-control pilot study aimed to explore potential personality differences between hypertensive and normotensive individuals from the perspective of the FFM and to identify whether different personality traits could potentially contribute as risk factors for hypertension. Our results revealed statistically significant differences between hypertensives and normotensives in the agreeableness and emotional stability dimensions, with a larger proportion of hypertensives scoring very low in agreeableness and low in emotional stability, in contrast to normotensives who presented high, respectively high and medium scores in agreeableness and emotional stability. Moreover, we found that low or very low emotional stability or higher neuroticism increases the odds of being hypertensive 4.51 times. Our findings are comparable to those in the literature, showing that individuals high in neuroticism, characterized by negative emotions and overreaction to stress, have a higher risk of developing hypertension. $(17,18)$ Neuroticism level is also known as a predictor of other health outcomes. High neuroticism was connected with premature mortality, increased risk of obesity and metabolic syndrome.(19) With regard to agreeableness, evidence suggests that hypertensive individuals present higher levels of Type A behaviour pattern than normotensives.(20) Hostility is the core feature of type A behavior (10) as well as a facet of agreeableness (18), indicating that there is a clear association between agreeableness and hypertension. Agreeableness describes a person's orientation toward others. Individuals scoring low on this dimension are less socially oriented, present a refractory and suspicious attitude towards others, are cynical, competitive, authoritarian, maintain a low opinion of human nature, present lack of altruism, hostility and irritability, need to oppose, to attack, to punish or to exclude those who are disliked or thought to be inferior.(21) Evidence shows that lower levels in agreeableness are also associated with higher mortality risk. $(18,22)$

We found no association between hypertension and conscientiousness, however growing evidence supports the relation of low conscientiousness with hypertension and other health outcomes such as diabetes, stroke and earlier mortality. $(23,24)$ Indeed, high neuroticism associated with low conscientiousness were shown as strong predictors of poor health outcomes.(25) The relations between other personality dimensions and health are less established.

Our pilot data can be used as a stepping stone to design large scale studies, to further investigate the relationship between personality and hypertension. The present management of hypertension is mainly pharmacotherapeutic with little evidence available to see if the management of different negative personality traits could have a preventive or therapeutic impact on the disease, which could prove particularly relevant for treatment resistant patients.

Finally, we recognize that there are limitations to this study that should be taken into account. First, this was a pilot study based in a single centre potentially introducing selection bias. Although it is worth noting that the recruitment centre receives patients from an extensive area and we also tried to limit selection bias by recruiting consecutive patients. In addition, the relatively small number of participants could have influenced the results. For example, we found no significant associations between some personality dimensions like conscientiousness and hypertension, although growing evidence supports the associations of these personality dimensions with health. Large, prospective, multi-centre studies are needed to fully investigate how different personality types could influence the development of hypertension and if different approaches to patients having a personality with risk (such as high neuroticism) could potentially benefit hypertension management or even play a role in prevention. Second, similarly to other studies, we have not excluded participants with other cardiovascular disorders. There are studies indicating that personality traits are associated with other CVD, especially with coronary heart disease. Therefore, the presence of individuals with coronary heart disease in hypertensive, respectively in the 


\section{PUBLIC HEALTH AND MANAGEMENT}

non-hypertensive group, could significantly influence the relationship between personality and hypertension.

\section{CONCLUSIONS}

Our results indicate that personality dimensions significantly vary between hypertensive and normotensive individuals, with low agreeableness and low emotiona stability/high neuroticism representing a potential risk factor for the development of hypertension. Large prospective studies further investigating this relationship and the value of integrating personality management in hypertension care would prove valuable.

\section{REFERENCES}

1. Kaplan NM. Systemic Hypertension: Mechanisms and Diagnosis. In: Bonow RO, Mann DL, Zipes DP, Libby P, editors. Braunwald's Heart Disease. A textbook of Cardiovascular Medicine. 9th ed. Philadelphia: Elsevier Saunders; 2012. p. 935-954.

2. Dorobantu M, Tautu OF, Dimulescu D, Sinescu C, Tatomir GP, Georgescu AC, et al. Perspectives on hypertension's prevalence, treatment and control in a high cardiovascular risk East European country: data from the SEPHAR III survey. Journal of Hypertension. 2018;36(3):690-700.

3. Cuffee Y, Ogedegbe C, Williams NJ, Ogedegbe G, Schoenthaler A. Psychosocial risk factors for hypertension: an update of the literature. Curr Hypertens Rep. 2014;16(10):483.

4. Williams B, Mancia G, Spiering W, Rosei AE, Azizi M, Burnier M, et al. $2018 \mathrm{ESH} / \mathrm{ESC}$ guidelines for the management of arterial hypertension: the Task Force for the Management of Arterial Hypertension of the European Society of Hypertension (ESH) and of the European Society of Cardiology (ESC). European Heart Journal. 2018;39:3021-3104.

5. Visseren FLJ, Mach F, Smulders YM, Carballo D, Koskinas CK, Back M, et al. 2021 ESC Guidelines on cardiovascular disease prevention in clinical practice: Developed by the Task Force for cardiovascular disease prevention in clinical practice with representatives of the European Society of Cardiology and 12 medical societies With the special contribution of the European Association of Preventive Cardiology (EAPC). European Heart Journal. 2021;42(34):3227-3337.

6. McCubbin JA, Nathan A, Hibdon MA, Castillo AV Graham JG, Switzer FS. Blood Pressure, Emotional Dampening, and Risk Behavior: Implications for Hypertension Development. Psychosomatic Medicine. 2018;80(6):544-550.

7. Cuevas AG, Williams DR, Albert MA. Psychosocial Factors and Hypertension: A Review of the Literature. Cardiology Clinics. 2017;35(2):223-230.

8. Costa PT, McCrae RR. The NEO personality inventory; 1985.

9. McCrae RR, John OP. An Introduction to the Five-Factor Model and Its Applications. 1992;60(2):175-215.

10. Matthews G, Deary IJ, Whiteman MC. Personality traits. Cambridge University Press; 2003.

11. Kupper N, Denollet J. Type D personality as a prognostic factor in heart disease: assessment and mediating mechanisms. J Pers Assess. 2007;89(3):265-276.

12. De Fruyt F, Denollet J. Type D personality: A Five-Factor Model Perspective. Psychol Health. 2002;17:671-683.

13. Kupper N, Denollet J. Type D Personality as a Risk Factor in Coronary Heart Disease: a Review of Current Evidence. Current Cardiology Reports. 2018;20(11):104.
14. Costa PT, Mac Crae RR. Neo personality inventoryrevised (NEO PI-R). Psychological Assessment Resources Odessa, FL; 1992.

15. Sava F. DECAS Personality inventory. Timişoara: Editura ArtPress; 2008.

16. Sava F, Popa RI. Personality types based on the Big Five model. A cluster analysis over the Romanian population. Cognition, Brain, Behavior. 2011;15(3).

17. Spiro A, Aldwin CM, Ward K, Mroczek DK. Personality and the incidence of hypertension among older men: Longitudinal findings from the Normative Aging Study. Health Psychol. 1995;14:563-569.

18. Turiano NA, Pitzer L, Armour C, Karlamangla A, Ryff CD, Mroczek DK. Personality trait level and change as predictors of health outcomes: Findings from a National Study of Americans (MIDUS). J Gerontol B Psychol Sci Soc Sci. 2012;67B:4-12.

19. Hampson SE, Friedman HS. Personality and health: A life span perspective. In: John OP, Robins R, Pervin L, editors. The handbook of personality. 3rd ed. New York: Guilford Press; 2008:770-794.

20. Sans J, Garcia-Vera MP, Magan I, Espinosa R, Fortun M. Differences in personality between sustained hypertension, isolated clinic hypertension and normotension. Eur J Pers. 2007;21:209-224.

21. Costa PT, Stone SV, McCrae RR, Dembroski TM, Williams RB. Hostility, Agreeableness-antagonism, and Coronary Heart Disease. Holistic Medicine (J Interprof Care). 1987;2:161-167.

22. Weiss A, Costa PT. Domain and Facet Personality Predictors of All-Cause Mortality Among Medicare Patients Aged 65 to 100. Psychosom Med. 2005;67:724733.

23. Bogg T, Roberts BW. Conscientiousness and HealthRelated Behaviors: A Meta-Analysis of the Leading Behavioral Contributors to Mortality. Psychol Bull. 2004;130:887-919.

24. Terracciano A, Strait J, Scuteri A, Meirelles O, Sutin AR, Tarasov $\mathrm{K}$ et al. Personality traits and circadian blood pressure patterns: A seven-year prospective study. Psychosom Med. 2014;76:237-243.

25. Friedman HS, Kern ML. Personality, well-being and health. Annu Rev Psychol. 2014;65:719-742. 